\title{
BMJ Open The involvement of physician assistants in inpatient care in hospitals in the Netherlands: a cost-effectiveness analysis
}

\author{
Marijke J C Timmermans, ${ }^{1,2}$ Geert T van den Brink, ${ }^{2}$ Anneke J A H van Vught, ${ }^{2}$ \\ Eddy Adang, ${ }^{3}$ Charles L H van Berlo, ${ }^{4}$ Kim van Boxtel, ${ }^{5}$ Weibel W Braunius, ${ }^{6,7}$ \\ Loes Janssen, ${ }^{8}$ Alyssa Venema, ${ }^{9}$ Frits J van den Wildenberg, ${ }^{10}$ Michel Wensing, ${ }^{1,11}$ \\ Miranda G H Laurant ${ }^{1,2}$
}

To cite: Timmermans MJC, van den Brink GT, van Vught AJAH, et al. The involvement of physician assistants in inpatient care in hospitals in the Netherlands: a cost-effectiveness analysis. BMJ Open 2017;7:e016405. doi:10.1136/ bmjopen-2017-016405

- Prepublication history and additional material are available. To view these files please visit the journal online (http://dx.doi org/10.1136/bmjopen-2017016405).

Received 11 February 2017 Revised 18 April 2017 Accepted 7 June 2017

CrossMark

For numbered affiliations see end of article.

Correspondence to Marijke J C Timmermans; Marijke.Timmermans@ radboudumc.nl

\section{ABSTRACT}

Objective To investigate the cost-effectiveness of substitution of inpatient care from medical doctors (MDs) to physician assistants (PAs).

Design Cost-effectiveness analysis embedded within a multicentre, matched-controlled study. The traditional model in which only MDs are employed for inpatient care (MD model) was compared with a mixed model in which, besides MDs, PAs are also employed (PA/MD model).

Setting 34 hospital wards across the Netherlands.

Participants 2292 patients were followed from admission until 1 month after discharge. Patients receiving daycare, terminally ill patients and children were excluded.

Primary and secondary outcome measures All direct healthcare costs from day of admission until 1 month after discharge. Health outcome concerned quality-adjusted life years (QALYs), which was measured with the EuroQol five dimensions questionnaire (EQ-5D).

Results We found no significant difference for QALY gain $(+0.02,95 \% \mathrm{Cl}-0.01$ to 0.05$)$ when comparing the PA MD model with the MD model. Total costs per patient did not significantly differ between the groups $(+€ 568$, $95 \% \mathrm{Cl}-€ 254$ to $€ 1391, \mathrm{p}=0.175)$. Regarding the costs per item, a difference of $€ 309$ per patient $(95 \% \mathrm{Cl} € 29$ to $€ 588, p=0.030$ ) was found in favour of the MD model regarding length of stay. Personnel costs per patient for the provider who is primarily responsible for medical care on the ward were lower on the wards in the PA/MD model $(-€ 11,95 \% \mathrm{Cl}-€ 16$ to $-€ 6, \mathrm{p}<0.01)$.

Conclusions This study suggests that the costeffectiveness on wards managed by PAs, in collaboration with MDs, is similar to the care on wards with traditional house staffing. The involvement of PAs may reduce personnel costs, but not overall healthcare costs. Trial registration number NCT01835444.

\section{BACKGROUND}

Because of an increased appreciation of continuity of care, pressure to deliver healthcare efficiently and local shortages of medical doctors (MDs), medical care for admitted patients is increasingly reallocated to
Strengths and limitations of this study

- This study increases the understanding of the implications of reallocating inpatient care from medical doctors to physician assistants on total healthcare costs as well as on resources uses.

- This study captured a large number of patients from 34 hospital wards, which cover both teaching and non-teaching hospitals and six different medical disciplines.

- The non-randomised character of this study implies an increased risk for confounding, which we accounted for in the multivariable analyses and subgroup analyses.

- Although we performed subgroup analyses, we cannot exclude that local differences like policies about quality of care and patient case-mix influence the results.

physician assistants (PAs). ${ }^{1-3} \mathrm{~A}$ PA is a health professional licensed to practise medicine in defined domains, with variable degrees of professional autonomy. ${ }^{4}$ PAs who provide medical care for admitted patients usually work in a team comprising both PAs and MDs (ie, residents, medical specialists or hospitalists).

Literature suggests that PAs add to the quality of care by increasing continuity for both patients and hospital staff. ${ }^{2}$ The turnover of house staff is traditionally high due to use of recent medical graduates who are planning to do fellowships and the mandatory rotational cycles. ${ }^{5}$ PAs generally do not rotate and constitute a factor of stability in the continually changing medical workforce. Previous studies show that quality of care for admitted patients delivered by a PA-based team is comparable to that of a resident-based team, and that patient evaluations are at least 
as good. ${ }^{6-10}$ Our own study showed similar quality and safety of care, but better patient experiences on wards with a PA-based team. ${ }^{11}$ Estimates of PA employment on costs vary across the conducted studies. ${ }^{9} 10$ These studies concerned one clinical discipline within one hospital, which reduces the generalisability of findings. Besides, all studies were conducted in the USA, where most hospitals involving PAs concern only acute care. In the Netherlands, most hospitals include both acute and chronic care under one roof. Given the outcomes of these studies and their limitations, we conducted a multicentre study that included PAs providing care to hospitalised patients including a range of clinical disciplines. This paper reports on the cost-effectiveness of substitution of inpatient care from MDs to PAs. Costs concerned all direct healthcare costs from day of admission until 1 month after discharge. Health outcome concerned quality-adjusted life years (QALYs), which is a composite measure of effectiveness consisting of quality of life and life years gained.

\section{METHODS}

\section{Study design}

This economic evaluation was performed alongside a multicentre, non-randomised, matched-controlled study, which was performed in the Netherlands. In this study, the care on hospital wards using a mixed 'PA/MD model' (intervention group) was compared with the care on wards using a solely 'MD model' (control group). ${ }^{12}$

\section{MD model}

In the MD model, only MDs provide medical care for admitted patients at a specific hospital department. Most of them are junior or senior residents. The resident is physically present at the department each weekday and is the first point of access to medical care during office hours (MR model). Their work includes daily clinical care and patient management. The residents are supervised by attending physicians. In some cases, especially in smaller hospitals where often no residents are employed, the medical specialists provide all medical care for the admitted patients (MS model). ${ }^{13}$

\section{PA/MD model}

In this model, the PAs who were employed on the wards are substitutes for the residents. Their tasks and responsibilities are largely comparable. PAs have the same authorisations as residents: they can make indications for treatment, perform predefined medical procedures and subscribe medication independently within their field of expertise. ${ }^{14}$ We included two different models within the intervention group: a model in which PAs collaborate with residents (mixed PA/MR model) and a model in which only PAs are the first point of access to medical care (PA model). In both models, the PAs as well as the residents were supervised by attending physicians.

Control wards were matched with the intervention wards on the basis of hospital type (ie, academic or non-academic) and medical specialty (ie, a range of surgical and medical specialties). No wards with general medicine were involved. Hospital wards were included in the intervention group if the PA covered at least $51 \%$ of the available ward care hours per week during day shifts on weekdays. Wards were included in the control group if exclusively MDs provided medical care. The primary analysis had patients' length of stay (LOS) as primary outcome. Further details of the study design have been described elsewhere. ${ }^{12}$ The economic analysis was conducted from a healthcare perspective, with a time frame from admission until 1 month after discharge.

\section{Study population}

This study focused on the patients admitted to the hospital wards. Exclusion criteria for patients were (1) younger than 18 years, (2) terminally ill and (3) receiving daycare. Daycare was defined as hospital admissions that were intended to last 24 hours or less (observation status).

\section{Health outcome}

The primary health outcome in this evaluation is the QALY. A QALY is a generic measure of disease burden. ${ }^{15}$ QALYs were derived using the EuroQoL-5D questionnaire (EQ-5D-3L), ${ }^{16}$ which is a widely used validated patient questionnaire comprising five domains: mobility, selfcare, usual activities, pain and anxiety/depression. Each domain has three possible levels indicating no problems, moderate problems or severe problems. The EQ-5D-3L was assessed at three time points: at admission, discharge and 1 month after discharge. We used the Dutch utility weight to calculate utilities. ${ }^{17}$

\section{Cost outcomes}

The primary cost outcome was the sum of direct costs associated with the principal admission and costs that occurred within 1 month after discharge that were potentially related to hospital admission. Resources used during admission were extracted in detail at an individual patient level from patient medical records, and included laboratory tests, diagnostic tests, medication and blood products. Also the frequency and type of consultations of healthcare suppliers and the number of days of unplanned stay at intensive care unit (ICU) were derived from the medical records. To minimise information bias, a random sample of $10 \%$ of the patient records per ward was reassessed by a second researcher, who was blinded to the results from the initial researcher. In case of an interrater agreement of less than $95 \%$, the records of the total sample were reassessed.

Personnel costs included the costs for the residents, PAs and medical specialist who were primarily employed for medical care for the admitted patients. Also the costs for supervision time by attending physicians were included. We measured the number of hours spent for medical ward care per professional by examination of work schedules. All MDs and PAs who had the primary task to provide medical care for admitted patients were asked to 
fill in their real work schedule during four fixed weeks: weeks 3, 7, 11 and 15 after the start of the inclusion of patients. Next, we divided the number of working hours by the number of patients for which they were in charge. The number of hours spent for supervision was derived from an online questionnaire. We asked each attending physician for the average number of hours they weekly spend for supervision. These hours were added up for all attending physicians of the department, and divided by the number of patients who were admitted at the ward.

Volumes that were measured between discharge and 1 month afterwards included days of unplanned readmission, number of presentations at emergency departments, number of contacts with a general practitioner and the required home care. These volumes were collected from a patient questionnaire that was sent 1 month after discharge. We chose for 1 month after discharge, as events happened after that period are less likely to be related to the initial admission period. ${ }^{18}$ Costs were calculated by multiplying the volumes of healthcare use with corresponding unit prices, derived from the Dutch Manual for Costing Research. ${ }^{19}$ All figures were related to the price level of the same year (ie, 2014). Details of the costs applied to units of resource use are provided in online supplementary file 1 .

\section{Sample size calculation}

Sample size calculation was based on LOS, which was the primary clinical outcome of the multicentre study. Results for LOS have been published elsewhere. ${ }^{11}$ The originally published sample size calculation ${ }^{12}$ was adjusted prior to start of data collection. ${ }^{20}$ To detect a relative difference in LOS of $20 \%$ between the 'PA/MD model' and 'MD model', assuming an average LOS of 6 days (SD 4.9), alpha of $5 \%$, power of $80 \%$ and an intracluster coefficient of 0.06 for patients on the same ward, 30 wards including 100 patients each were required. Taking into account an expected dropout of a maximum of two matched pairs, 34 wards (17 in each arm) with each 100 patients were required. In case of no dropout, 50 patients per ward would be sufficient.

\section{Data analysis}

We used descriptive analyses with counts (and proportions) or means (with SDs) to describe baseline characteristics, effects and costs. The a priori planned analysis was a comparison between the intervention and control group on incremental costs and incremental effects. The incremental effects were analysed using a linear mixed model approach with the QALY score as dependent variable and group and baseline QALY as independent variables, taking clustering of patients within wards into account. If similar effects on the QALY in both groups were found, a cost-minimisation approach was performed by comparing differences in costs between groups using a linear mixed model approach accounting for clustering and applying bootstrapping (200 times) to create bias-corrected 95\% CIs around the coefficients of the independent variables.
A total of 50-200 replications are generally adequate for estimates of SE. ${ }^{21}$ Multivariable models were constructed to adjust for potential confounders. We took matching into account by adding covariables for the matching variables.

Missing data were imputed via multiple imputations, which was embedded within the statistical package. To explore uncertainty around costing assumptions (ie, cost prices and salary), sensitivity analysis was conducted on the range of extremes. Imputation models for all cost categories and utility scores were then redone accounting for changes in the sensitivity analysis. To explore heterogeneity within the results, post-hoc subgroup analyses were performed for each submodel of medical ward care, that is (1) the MS model: medical specialists are in charge of all admitted patients; (2) MR model: residents or junior doctors are in charge of all admitted patients; (3) mixed PA/MR model: both residents and PAs are in charge of the admitted patients; and (4) PA model: PAs are in charge of all admitted patients. ${ }^{13}$ All analyses were carried out with Stata V.11.2. $p$ Value was set at 0.05 to indicate statistical significance.

\section{Ethical considerations}

Ethical approval was received from the Research Ethics Committee of the Radboud university medical center, Nijmegen (registration number: 2012/306); the committee judged that ethical approval was not required under Dutch Law. All data were handled strictly confidential and written informed consent was obtained from all patients.

\section{RESULTS}

Between April 2013 and May 2015, we included 1021 patients spread over 17 hospital wards in the intervention group, and 1286 patients spread over 17 hospital wards in the control group (figure 1). In total, 23 hospitals across the Netherlands were involved. More patients in the intervention group were acutely admitted (59\% vs $44 \%$ in the control group, $\mathrm{p}<0.01)$. Also medical specialty, hospital type, primary diagnosis and discharge destination differed significantly between the groups (table 1).

\section{Health outcomes}

We had complete QALY data for 779 patients in the intervention group $(76 \%)$ and 982 patients in the control group $(76 \%)$. Utilities related to the three time points and QALYs are outlined in table 2. The EQ-5D utilities did not statistically significantly differ between the study arms at baseline and throughout the study. At discharge and 1 month after discharge, the mean difference in EQ-5D utility was -0.01 ( $95 \% \mathrm{CI}-0.06$ to $0.04, \mathrm{p}=0.634)$ and -0.04 ( $95 \%$ CI -0.09 to $0.02, \mathrm{p}=0.178$ ), respectively, corrected for baseline utility. Similarly, the difference in QALY gain was not statistically significant during admission nor after discharge. 


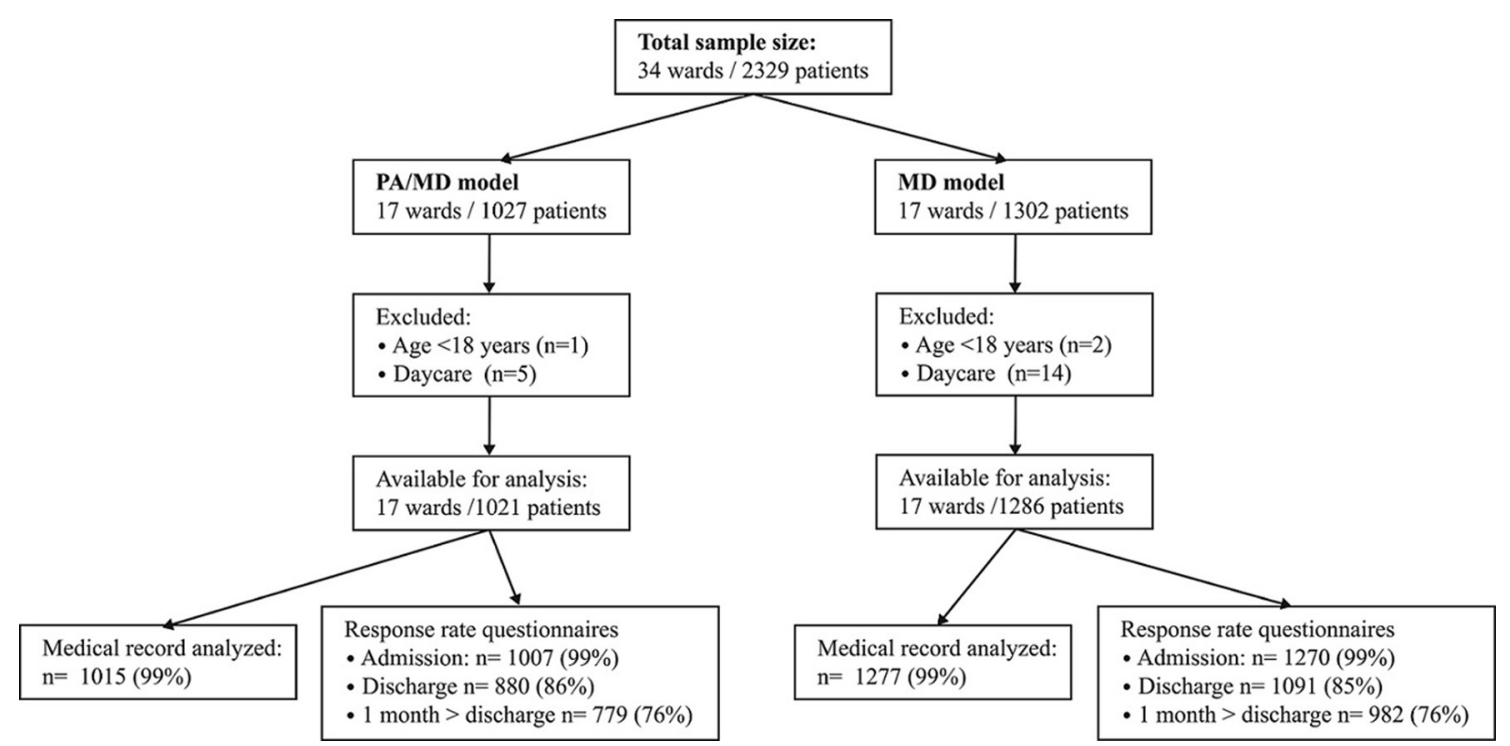

Figure 1 Flow chart of patients. MD, medical doctor; PA, physician assistant.

\section{Resource use and costs}

Ninety-nine per cent of all patient records were assessed. Item missing varied from $2 \%$ (unplanned transfer to ICU) to $9 \%$ (use of blood products). Resource use after discharge was derived from the questionnaire, which was sent to the patient 1 month after discharge. The response rate on this questionnaire was $76 \%$ in both study arms. Resources used during the period from admission until 1 month after discharge are summarised in online supplementary file 2 .

Table 3 outlines the total costs per patient and costs per item. The mean total costs per patient in the intervention group did not significantly differ from the mean costs per patient in the control group: mean difference was $€ 568$ (95\% CI $-€ 254$ to $€ 1391, \mathrm{p}=0.175$ ). Regarding the costs per item, we found significant differences of $€ 309$ per patient ( $95 \% \mathrm{CI} € 29$ to $€ 588, \mathrm{p}=0.030$ ) regarding LOS in favour of the MD model. Personnel costs for the PA or MD who is primarily responsible for the medical care on the ward were significantly lower on the wards in the PA/MD model: mean difference $-€ 11(95 \%$ CI $-€ 16$ to $-€ 6, \mathrm{p}<0.01)$ per patient. Costs for supervision by the staff physicians were significantly higher in the PA/MD model: mean difference $€ 43(95 \%$ $\mathrm{CI} € 39$ to $€ 47, \mathrm{p}<0.01)$. Since the MD model also incorporates wards with only medical specialists, supervision is not applicable for these wards. To rule out this distortion, we performed an additional analysis in which we excluded the four wards with only medical specialists. This resulted in an opposite difference: costs for supervision were now significantly lower for the $\mathrm{PA} / \mathrm{MD}$ model compared with the MD models: mean difference $-€ 11$ (95\% CI $-€ 16$ to $-€ 6, \mathrm{p}<0.01)$.

Sensitivity analyses on the range of extremes did not change these results of the total costs and costs per item substantially (data not shown, but can be provided on re142013quest).

\section{Subgroup analyses}

Results for the analyses per submodel of medical ward care are shown in table 4 . The mean total costs per patient did not significantly differ among the submodels. Costs for LOS were on average $€ 465$ per patient (95\% CI -920 to $-10, \mathrm{p}=0.045)$ lower in the MS model than in the mixed PA/MR model. The other models did not significantly differ from each other. Personnel costs for the provider who is primarily responsible for the medical care on the ward were significantly highest in the MS model (mean $€ 129$ (SD €37)) and lowest in the PA model (mean €51 $(\mathrm{SD} € 3)$ ). Costs for supervision were significantly highest in the MR model (mean $€ 178$ ( $\mathrm{SD} € 79)$ ) and lowest in the PA model (mean €121 ( $\mathrm{SD} € 59)$ ). We also found significant differences regarding costs for blood products and required home care: these were highest in the PA model.

\section{DISCUSSION}

This study aimed to determine the cost-effectiveness of substitution of inpatient care from MDs to PAs. No significant difference between the two study arms was found on QALY and total costs. Explorative analyses showed a significant difference in costs for LOS in favour of the MD model, and significant differences regarding personnel costs in favour of the PA/MD model.

To our knowledge, this is the first multicentre study that investigated the cost implications of reallocating inpatient care from MDs to PAs. A few single-centred studies have compared costs of non-acute inpatient care delivered by a PA-based team with care delivered by a resident-based team. ${ }^{9}{ }^{10}$ These studies did not measure QALYs. Results regarding total costs were mixed. Roy et a $\hat{\theta}$ reported that the care by the PA-based team was associated with lower total costs per patient, while Singh $e t a l^{10}$ reported similar costs between the study arms. These studies can however 
Table 1 Baseline characteristics of patients

\section{Baseline characteristics}

Medical specialty, $\mathrm{n}(\%)$

\begin{tabular}{lc} 
Surgery & $601(59 \%)$ \\
Gastroenterology & $102(10 \%)$ \\
Pulmonology & $91(9 \%)$ \\
Cardiology & $101(10 \%)$ \\
Orthopaedics & $103(10 \%)$ \\
ENT, head and neck oncology surgery & $23(2 \%)$ \\
Hospital type, n(\%) & \\
\hline Teaching & $552(54 \%)$ \\
$\quad$ Academic & $23(2 \%)$ \\
$\quad$ Non-academic & $529(52 \%)$ \\
Non-teaching & $469(46 \%)$ \\
Gender, male, n(\%) & $524(53 \%)$ \\
Age, years, mean \pm SD & $64 \pm 16$ \\
Major diagnoses, $n(\%)$ & \\
\hline Digestive system & $204(20 \%)$ \\
Circulatory system & $158(16 \%)$ \\
Neoplasms & $108(11 \%)$ \\
Musculoskeletal system and connective tissue & $120(12 \%)$ \\
Injury and poisoning & $135(13 \%)$ \\
Infectious and parasitic diseases & $59(6 \%)$ \\
Respiratory system & $51(5 \%)$ \\
Symptoms & $61(6 \%)$
\end{tabular}

Charlson index for comorbidity score, mean \pm SD (\% with $1.1 \pm 1.8(43 \%)$ score $\geq 1$ )

Highest education, $\mathrm{n}(\%)$

Low
Middle
High

Ethnicity, Dutch, $\mathrm{n}(\%)$

Marital status, $\mathrm{n}(\%)$

$\begin{array}{lr}\text { No partner } & 136 \\ \text { Partner } & 730 \\ \text { Widow } & 119 \\ \text { Smoking status, } \mathrm{n}(\%) & \end{array}$

No, never smoked
No, but ever smoked

Yes, still smoking

Body mass index (mean \pm SD)

Number of hospitalisations for same problem, $\mathrm{n}(\%)$

1 hospitalisation

$>1$ hospitalisation

Type of admission, $\mathrm{n}(\%)$

Elective

Urgent

\section{PA/MD model $(n=1021)$}

(n)

\section{MD model $(n=1286)$}

p Value

$<0.01$

696 (54\%)

$181(14 \%)$

107 (8\%)

$124(10 \%)$

$100(8 \%)$

$78(6 \%)$

$<0.01$

$709(55 \%)$

$78(6 \%)$

$631(49 \%)$

$577(45 \%)$

$682(54 \%)$

0.47

$63 \pm 15$

0.11

$<0.01$

247 (19\%)

274 (22\%)

$195(15 \%)$

$119(9 \%)$

$80(6 \%)$

$81(6 \%)$

$75(6 \%)$

$87(7 \%)$

$\begin{array}{ll}1.1 \pm 1.8(44 \%) & 0.65 \\ 0.66\end{array}$

0.15

$371(38 \%)$

$422(34 \%)$

$489(40 \%)$

$328(27 \%)$

$233(24 \%)$

$1212(98 \%)$

0.15

$976(99 \%)$

$136(14 \%)$

0.29

$730(74 \%)$

$119(12 \%)$

$949(77 \%)$

$125(10 \%)$

$325(33 \%) \quad 385(31 \%)$

$494(48 \%) \quad 626(50 \%)$

$174(17 \%) \quad 230(19 \%)$

$27 \pm 5$

$27 \pm 5$

0.79

0.65

580 (59\%)

693 (56\%)

$403(41 \%)$

$540(44 \%)$

402 (41\%)

687 (56\%)

$588(59 \%)$ 


\begin{tabular}{|c|c|c|c|}
\hline Baseline characteristics & PA/MD model $(n=1021)$ & MD model $(n=1286)$ & p Value \\
\hline Discharge destination, n(\%) & & & $<0.01$ \\
\hline Home & 765 (90\%) & $965(92 \%)$ & \\
\hline Hospital & $12(1 \%)$ & $30(3 \%)$ & \\
\hline Nursing home/rehabilitation centre/hospice & $56(7 \%)$ & $28(3 \%)$ & \\
\hline Family relative & $18(2 \%)$ & $25(2 \%)$ & \\
\hline
\end{tabular}

Numbers may not add up to the total because of missing values.

ENT, ear, nose, throat; MD, medical doctor; PA, physician assistant.

hardly be compared with our study because different methods to estimate costs were used and the settings were different. In addition, most of these studies compared a hospitalist/PA model with the traditional resident-based model, while hospitalists were not part of the models we used. $^{13}$

Our previous analysis showed increased provider continuity on the ward with the presence of a PA. ${ }^{13}$ This study shows that this increased continuity did not cause a decrease in costs, especially because of the higher costs for LOS. Subgroup analysis showed that costs for LOS were especially higher when compared with the model in which only medical specialists were involved. Costs did not significantly differ between the PA models and the model that involves only residents (MR model). An explanation for the lower costs for LOS in the MS model might be that the medical specialists have more work experience. The PA profession is relatively new; most of them have a short time of experience compared with medical specialists. ${ }^{13}$ Over time the clinical experience of PAs will become larger, which may lead to lower costs. Besides, we cannot exclude the possibility that the lower LOS indicates that the patients who were included in the MS model were overall less complex than the patients in the other models. Although we have adjusted for relevant confounders in the multivariable analysis, it is not possible to perfectly adjust for the complexity of the patient in non-randomised comparisons.

Personnel costs for the provider who is primarily responsible for the medical care on the ward were significantly lower on the wards with the PA/MD model when compared with the MD model. Subgroup analysis showed highest costs on the wards with only medical specialists. This can be explained by the significantly higher salary. Besides, we found lower costs on wards with the PA model when compared with the model that involves only residents. Since in the Netherlands the salary of PAs is comparable to the salary of residents (online supplementary file 1), the significant difference can be explained by our finding that on the wards with the PA/MD model, less time was spent per patient (online supplementary file 2 ). This is probably caused by the finding of our previous study that PAs spend less time on indirect inpatient care than residents do. ${ }^{13}$ A hypothesis is that since PAs tend to work for a longer time on the hospital ward, they might be more familiar with the clinical protocols and the procedures, for example when requesting diagnostic tests and consultation of other physicians. Also the increased provider continuity might lead to more efficient care. ${ }^{13}$

In our initial analysis, costs for supervision were significantly higher in the PA/MD model when compared with the MD model. However, this finding was biased by the wards with only medical specialists, since supervision was not applicable for these wards. Costs for supervision were higher on the wards with the mixed PA/MR model and the MR model when compared with the PA model. An explanation might be the fact that the PAs in the PA model have more work experience than the PAs and residents in the other models. ${ }^{13}$ An alternative hypothesis is that the difference is caused by the teaching culture of

Table 2 Utilities at admission, discharge and 1 month after discharge, and QALY gained

\begin{tabular}{|c|c|c|c|c|}
\hline Outcome & $\begin{array}{l}\text { PA/MD model }(n=1015) \\
\text { mean }(S D)^{*}\end{array}$ & $\begin{array}{l}\text { MD model }(n=1277) \\
\text { mean }(S D)^{\star}\end{array}$ & $\begin{array}{l}\text { Difference mean } \\
(95 \% \mathrm{Cl})\end{array}$ & p Value \\
\hline \multicolumn{5}{|l|}{$E Q-5 D$} \\
\hline Discharge & $0.71(0.22)$ & $0.72(0.23)$ & $-0.01(-0.06$ to 0.04$)$ & 0.634 \\
\hline One month after discharge & $0.75(0.23)$ & $0.78(0.22)$ & $-0.04(-0.09$ to 0.02$)$ & 0.178 \\
\hline
\end{tabular}

*Values are summary estimates obtained by multiple imputation.

†Difference in QALY between 1 month after discharge and discharge, adjusted for baseline utility.

EQ-5D, EuroQol five dimensions questionnaire; MD, medical doctor; PA, physician assistant; QALY, quality-adjusted life years. 
Table 3 Total costs per patient and costs per item $(€)$

\begin{tabular}{|c|c|c|c|c|}
\hline & $\begin{array}{l}\text { PA/MD model } \\
(n=1015)\end{array}$ & $\begin{array}{l}\text { MD model } \\
(n=1277)\end{array}$ & Difference† & \\
\hline Item & Mean (SD)* & Mean (SD)* & Mean $(95 \% \mathrm{Cl})$ & p Value \\
\hline \multicolumn{5}{|l|}{ Costs associated with principal admission } \\
\hline Length of stay & $1780(1811)$ & $1421(1210)$ & 309 (29 to 588$)$ & 0.030 \\
\hline Non-elective transfer to ICU & $333(3267)$ & $182(1761)$ & $105(-262$ to 473$)$ & 0.575 \\
\hline Laboratory tests & $107(168)$ & $99(136)$ & $19(-16$ to 44$)$ & 0.366 \\
\hline Diagnostic tests & $163(229)$ & $154(235)$ & $-1(-44$ to 42$)$ & 0.970 \\
\hline Blood products & $31(122)$ & $36(117)$ & $-12(-37$ to 14$)$ & 0.371 \\
\hline \multicolumn{5}{|l|}{ Consultation with healthcare suppliers } \\
\hline $\begin{array}{l}\text { PA/MD who is primarily responsible for medical } \\
\text { care }\end{array}$ & $71(29)$ & $103(44)$ & $-31(-33$ to -28$)$ & $<0.01$ \\
\hline Supervision by staff physician & $156(93)$ & $129(104)$ & 43 (39 to 47$)$ & $<0.01$ \\
\hline Exclusion of wards with staff physicians only & $156(93)$ & $173(77)$ & $-11(-16$ to -6$)$ & $<0.01$ \\
\hline \multicolumn{5}{|l|}{ Costs occurred during first month after discharge } \\
\hline Presentation at emergency department & $108(182)$ & $114(298)$ & $-13(-45$ to 20$)$ & 0.448 \\
\hline Non-elective readmission & $456(1333)$ & $421(1142)$ & $1(-89$ to 92$)$ & 0.977 \\
\hline Contact with general practitioner & $55(73)$ & $53(70)$ & $0(-7$ to 7$)$ & 0.923 \\
\hline Required home care & $121(248)$ & $98(214)$ & $11(-9$ to 30$)$ & 0.275 \\
\hline
\end{tabular}

*Values are summary estimates obtained by multiple imputation.

†Difference in mean costs per patient in the PA/MD group minus the MD group with bootstrapped $95 \% \mathrm{Cl}$, adjusted for medical specialty, hospital type, diagnosis, comorbidities, type of admission and discharge destination.

ICU, intensive care unit; MD, medical doctor; PA, physician assistant.

the wards. Eighty-three per cent of all included wards with a mixed PA/MR model and $69 \%$ of all wards with the MR model are from teaching centres, while none of the wards with the PA model are. ${ }^{13}$ As a consequence, there might be more consultation between professionals and more emphasise on education, which could be included in the supervision hours.

This study suggests that the cost-effectiveness of inpatient care delivered by a PA-based team is comparable to that of residents-based teams. This does not confirm the findings from qualitative studies, in which medical specialists experienced an increased efficiency after employing PAs. ${ }^{52}{ }^{23}$ However, the effectiveness that was experienced by the interviewed providers in our own qualitative study was based on items that were not in the scope of this quantitative research. ${ }^{5}$ Several interviewees experienced increased effectiveness because the PA performs additional tasks that were normally the responsibility of the staff physicians or residents, like integrating newly employed doctors, performing specific (complex) medical procedures, providing education or conducting quality projects. As a consequence, staff physicians and residents can be employed more effectively in, for example, providing outpatient care or conducting surgery. Besides, residents experience increased effectiveness because they have more time to focus on the needs for their own education.

This economic evaluation was conducted from a healthcare perspective. The societal perspective was not taken into account. For example, educational costs for PA students are thought to be lower than educational costs for medical students, since the vocational training programmes take 2.5 and 6 years, respectively. Exact costs for training PA students are however hard to determine, because Dutch PA students have already obtained a healthcare-related bachelor's degree of 4 years and have at least 2 years of clinical work experience in the healthcare domain. ${ }^{24}$ Besides, since the PA education is a shortened form of the traditional medical education, it is thought that policy makers can respond quicker on the frequently changing demand for medical professionals within healthcare organisations. Another value from the social perspective might be that becoming a PA is an interesting opportunity for nurses and other 


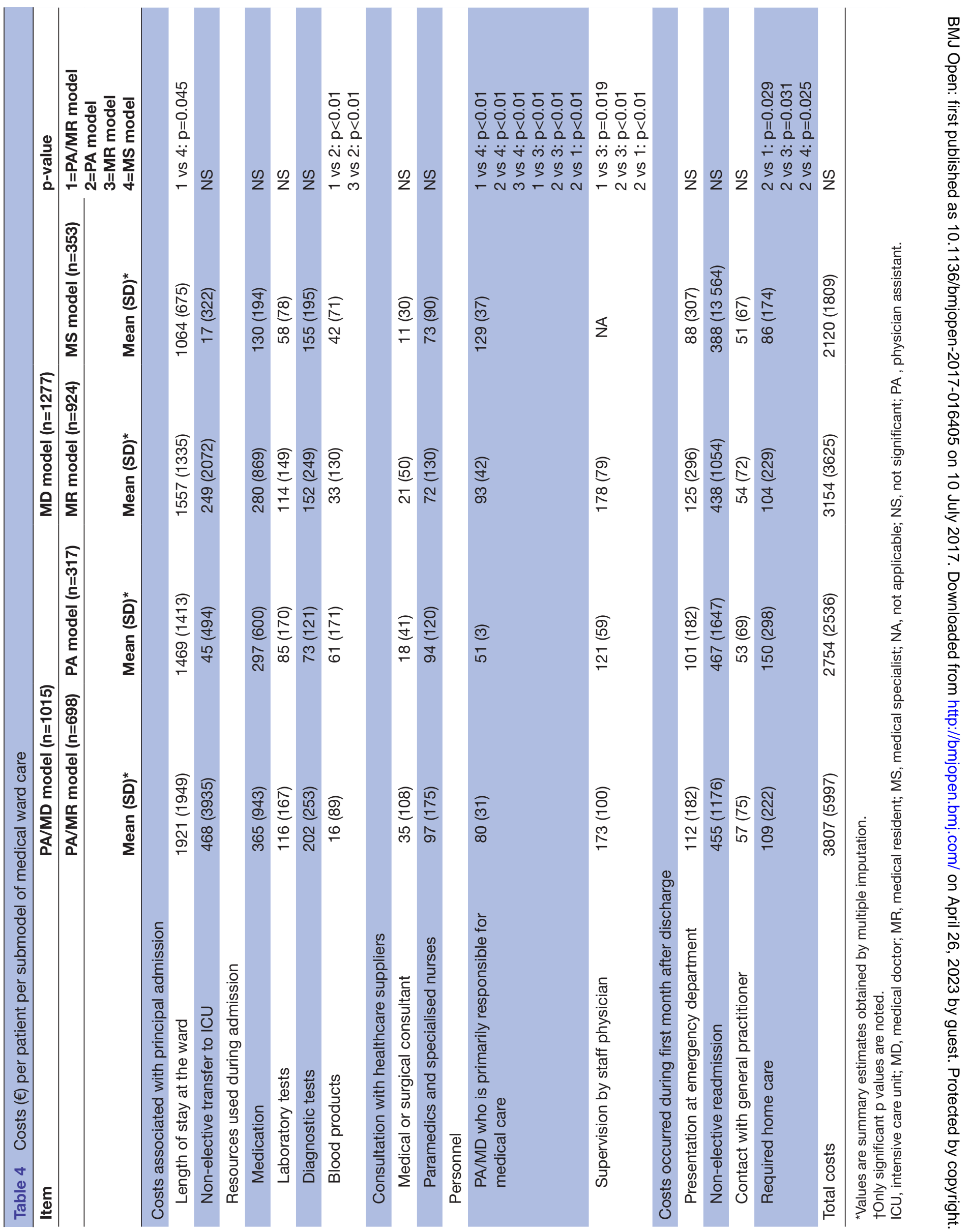


healthcare providers wanting to advance their career. ${ }^{25} 26$ As a consequence, motivated employees can be saved for the healthcare workforce.

Several strengths and limitations have to be mentioned. A strength is the multicentre design, which increases the generalisability of our findings. We included a broad range of clinical disciplines from different types of hospitals. A limitation is the non-randomised design. Different from other countries, the Dutch PA programmes incorporate a dual work-education model, which means that students are employed within a particular medical specialty from the day of their enrolment in the master's PA programme. ${ }^{24}{ }^{27}$ After graduation, the majority continue their employment at the same department. The suggestion of randomly relocating the graduated PA to another hospital ward could lead to resistance among the staff physicians who put considerable effort in the training. The non-randomised character of this study does imply an increased risk for confounding, which we accounted for in the multivariable analyses. Besides, we tried to reduce heterogeneity within our data by conducting subgroup analyses for the four models for medical ward care separately. However, we cannot exclude that there are still local differences like policies about quality of care and patient case-mix, which still influence our results. Besides, the results of the subgroup analyses should be interpreted with caution because of the low numbers of patients per subgroup.

\section{CONCLUSION}

This study suggests that the cost-effectiveness on wards managed by PAs, in collaboration with MDs, is similar to the care on wards with traditional house staffing by MDs only. The implementation of PAs may reduce personnel costs, but not overall healthcare costs.

\section{Author affiliations}

${ }^{1}$ Radboud university medical center, Radboud Institute for Health Sciences, Scientific Center for Quality of Healthcare (IQ Healthcare), Nijmegen, The Netherlands

${ }^{2}$ HAN University of Applied Sciences, Faculty of Health and Social Studies, Nijmegen ${ }^{3}$ Department of Health Evidence, Radboud university medical center, Radboud Institute for Health Sciences, Nijmegen, The Netherlands

${ }^{4}$ Department of Surgery Venlo, VieCuri Medical Center Noord-Limburg, Venlo, The Netherlands

${ }^{5}$ Department of Gastroenterology, Jeroen Bosch Hospital, Den Bosch, The Netherlands

${ }^{6}$ Department of Head and Neck Surgical Oncology, UMC Cancer Center, University Medical Center Utrecht, Utrecht, The Netherlands

${ }^{7}$ Department of Otorhinolaryngology, University Medical Center Utrecht, Utrecht, The Netherlands

${ }^{8}$ Department of Orthopaedics, VieCuri Medical Center Noord-Limburg, Venlo, The Netherlands

${ }^{9}$ Department of Surgery, Elisabeth-Tweesteden Hospital, Tilburg, The Netherlands ${ }^{10}$ Department of Surgery, Canisius Wilhelmina Hospital, Nijmegen, The Netherlands

${ }^{11}$ Department of General Practice and Health Services Research, Heidelberg University Hospital, Heidelberg, Germany

Acknowledgements We thank all professionals of the 34 Dutch hospital wards for participating in the study: Bravis Hospital, Department of Orthopaedics; Canisius Wilhelmina Hospital, Department of Surgery; Canisius Wilhelmina Hospital, Department of Gastroenterology; De Tjongerschans Hospital, Department of
Surgery; Elisabeth Hospital, Department of Surgery; Elkerliek Hospital, Department of Surgery; Elkerliek Hospital, Department of Cardiology; Gelre Hospital, Department of Pulmonology; Haga Hospital, Department of Surgery; Hospital Gelderse Vallei, Department of Surgery; Jeroen Bosch Hospital, Department of Surgery; Jeroen Bosch Hospital, Department of Gastroenterology; Koningin Beatrix Hospital, Department of Surgery; Laurentius Hospital, Department of Surgery; Laurentius Hospital, Department of Gastroenterology; Medical Center Haaglanden, Department of Surgery; Orbis Medical Center, Department of Surgery; Radboud university medical center, Department of ENT, Head and Neck Surgical Oncology; Reinier de Graaf Gasthuis, Department of Surgery; Rijnstate Hospital, Department of Gastroenterology; Scheper Hospital, Department of Surgery; Slingeland Hospital, Department of Surgery; TweeSteden Hospital, Department of Pulmonology; Van Weel Bethesda Hospital, Department of Surgery; UMC Cancer Center, University Medical Center Utrecht, Department of Head and Neck Surgical Oncology; VieCuri Medical Center Noord-Limburg, Department of Surgery; VieCuri Medical Center Noord-Limburg, Department of Cardiology; VieCuri Medical Center NoordLimburg, Department of Pulmonology; and VieCuri Medical Center Noord-Limburg, Department of Orthopaedics. Second, we thank Irma Maassen and Yvonne Peters (research assistants, Radboud university medical center, Scientific Center for Quality of Healthcare) for executing many logistical procedures. Third, we thank Reinier Akkermans (Radboud university medical center, Scientific Center for Quality of Healthcare), statistical expert, for his advice in data analysis.

Contributors MGHL and MJCT are responsible for the design of the study, with comments of AJAHvV, MW, EA and GTvdB. MJCT is responsible for the data collection and data management, with direct supervision and feedback from MGHL. MJCT and EA conducted the data analyses. CLHvB, KvB, WWB, LJ, AV and FJvdW were involved in the data collection of the study. MJCT wrote the first draft of the manuscript, and all other authors reviewed this critically. All authors read and approved the final manuscript.

Funding This work was funded by the Netherlands Organisation for Health Research and Development (ZonMw), grant number 171202006.

Competing interests None declared.

Patient consent Obtained.

Ethics approval The Research Ethics Committee of the Radboud university medical center, Nijmegen.

Provenance and peer review Not commissioned; externally peer reviewed.

Data sharing statement Data files are available from the authors on reasonable request.

Open Access This is an Open Access article distributed in accordance with the Creative Commons Attribution Non Commercial (CC BY-NC 4.0) license, which permits others to distribute, remix, adapt, build upon this work non-commercially, and license their derivative works on different terms, provided the original work is properly cited and the use is non-commercial. See: http://creativecommons.org/ licenses/by-nc/4.0/

(C) Article author(s) (or their employer(s) unless otherwise stated in the text of the article) 2017. All rights reserved. No commercial use is permitted unless otherwise expressly granted.

\section{REFERENCES}

1. Cawley JF, Hooker RS. Physician assistants in American medicine: the half-century mark. Am J Manag Care 2013;19:e333-41.

2. Ford WT, Britting LL. Nonphysician providers in the hospitalist model: a prescription for change and a warning about unintended side effects. J Hosp Med 2010;5:99-102.

3. Hartsell $Z$. The emerging role of PAs in the hospitalist movement. JAAPA 2007;20:10.

4. Merkle F, Ritsema TS, Bauer S, et al. The physician assistant: Shifting the Paradigm of European medical practice? HSR Proc Intensive Care Cardiovasc Anesth 2011;3:255-62.

5. Timmermans MJ, van Vught AJ, Maassen IT, et al. Determinants of the sustained employment of physician assistants in hospitals: a qualitative study. BMJ Open 2016;6:e011949.

6. Dhuper S, Choksi S. Replacing an academic internal medicine residency program with a physician assistant--hospitalist model: a comparative analysis study. Am J Med Qual 2009;24:132-9.

7. Oswanski MF, Sharma OP, Raj SS. Comparative review of use of physician assistants in a level I trauma center. Am Surg 2004;70:272. 
8. Nishimura RA, Linderbaum JA, Naessens JM, et al. A nonresident cardiovascular inpatient service improves residents' experiences in an academic medical center: a new model to meet the challenges of the new millennium. Acad Med 2004;79:426-31.

9. Roy CL, Liang CL, Lund M, et al. Implementation of a physician assistant/hospitalist service in an academic medical center: impact on efficiency and patient outcomes. J Hosp Med 2008;3:361-8.

10. Singh S, Fletcher KE, Schapira MM, et al. A comparison of outcomes of general medical inpatient care provided by a hospitalist-physician assistant model vs a traditional resident-based model. J Hosp Med 2011;6:122-30.

11. Timmermans MJC, Van Vught A, Peters YAS, et al. The impact of the implementation of physician assistants in inpatient care: a multicenter matched-controlled study. Submitted.

12. Timmermans MJ, van Vught AJ, Wensing M, et al. The effectiveness of substitution of hospital ward care from medical doctors to physician assistants: a study protocol. BMC Health Serv Res 2014;14:43.

13. Timmermans MJ, van Vught AJ, Van den Berg M, et al. Physician assistants in medical ward care: a descriptive study of the situation in the Netherlands. J Eval Clin Pract 2016;22:395-402.

14. De Bruijn-Geraets DP, Van Eijk-Hustings YJ, Vrijhoef HJ. Evaluating newly acquired authority of nurse practitioners and physician assistants for reserved medical procedures in the Netherlands: a study protocol. J Adv Nurs 2014;70:2673-82.

15. Drummond MF, Sculpher MJ, Torrance GW, et al. Methods for the economic evaluation of health care programmes. Third ed, 2005.

16. EuroQol Group. EuroQol--a new facility for the measurement of health-related quality of life. Health Policy 1990;16:199-208.

17. Lamers LM, Stalmeier PF, McDonnell J, et al. Measuring the quality of life in economic evaluations: the dutch EQ-5D tariff. Ned Tijdschr Geneeskd 2005;149:1574-8.
18. Halfon P, Eggli Y, van Melle G, et al. Measuring potentially avoidable hospital readmissions. J Clin Epidemiol 2002;55:573-87.

19. Hakkaaer- van Roijen L, Tan S. Bouwmans CAM: handleiding voor Kostenonderzoek. Methoden en standaard kostprijzen voor economische evaluaties in de gezondheidszorg. Rotterdam: Health care Insurance Council, 2015.

20. Timmermans MJ, van Vught AJ, Wensing M, et al. Erratum to: The effectiveness of substitution of hospital ward care from medical doctors to physician assistants: a study protocol. BMC Health Serv Res 2016;16:115.

21. Mooney CZ, Duval RD. Bootstrapping: a nonparametric approach to statistical inference. London: SAGE Publications, 1993.

22. Taylor MT, Wayne Taylor D, Burrows K, et al. Qualitative study of employment of physician assistants by physicians: benefits and barriers in the Ontario health care system. Can Fam Physician 2013;59:e507-13.

23. White $\mathrm{H}$, Round JE. Introducing physician assistants into an intensive care unit: process, problems, impact and recommendations. Clin Med 2013;13:15-18.

24. Spenkelink-Schut G, ten Cate OTJ, Kort HSM. Training the Physician Assistant in the Netherlands. The Journal of Physician Assistant Education 2008;19:46-53.

25. Niezen MG, Mathijssen JJ. Reframing professional boundaries in healthcare: a systematic review of facilitators and barriers to task reallocation from the domain of medicine to the nursing domain. Health Policy 2014;117:151-69.

26. Zwijnenberg NC, Bours GJ. Nurse practitioners and physician assistants in Dutch hospitals: their role, extent of substitution and facilitators and barriers experienced in the reallocation of tasks. $J$ Adv Nurs 2012;68:1235-46.

27. Hooker RS, Kuilman L. Physician assistant education: five countries. $J$ Physician Assist Educ 2011;22:53-8. 